\title{
A Web-based study of cerebral asymmetry for perception of emotion
}

\author{
LINDA RUECKERT \\ Northeastern Illinois University, Chicago, Illinois
}

\begin{abstract}
Many studies have examined cerebral asymmetry via lateralized presentation of stimuli using specialized software. Because these studies typically require extensive control over variables such as stimulus exposure duration, size, location, and so forth, they are not easily transferred to the World Wide Web. The present study replicated a free-vision, chimeric faces task via the Internet, using HTML and JavaScript. Previous results had shown that right-handed participants tend to perceive a chimeric face with a smile to their (the viewers') left as happier than its mirror image. That result was replicated in the Web-based study, and the leftward bias was actually significantly greater than that found using the traditional pencil-and-paper version of the task. Possible reasons for this difference are discussed.
\end{abstract}

The many potential benefits of using the World Wide Web to conduct research have been discussed extensively (Gosling, Vazire, Srivastava, \& John, 2004; Kraut et al., 2004; Reips, 2002). Most of the benefits center around the increase in external validity that results from the increase in sample size and diversity. These benefits must be weighed against the costs that arise from lack of control over stimuli, testing environment, and subject selection. Nevertheless, a large number of studies have used the Web to administer surveys and report results virtually identical to those found using the more traditional pencil-and-paper surveys. Most of these have been within the field of social and cognitive psychology, but far fewer have been reported in the field of neuropsychology. This is likely because neuropsychological studies typically require relatively greater control over the conditions of stimulus presentation and researchers in these fields often place less emphasis on external validity. For example, studies of cerebral asymmetry in normal participants are often conducted using lateralized presentation of stimuli via computers running specialized software. Because these studies typically require a great deal of control over variables such as stimulus size, location, exposure duration, and so forth, they are not as easy to translate into Web-based experiments as are surveys. One study that did successfully adapt a lateralization task to the Web was that of McGraw, Tew, and Williams (2000), who found a significant right visual field advantage in reaction time to verbal stimuli. However, that study utilized an Authorware program that required participants to download a plug-in for their browser before partici-

I thank Tony Carlsen for technical assistance and Jerre Levy and two anonymous reviewers for helpful comments on an earlier version of the manuscript. Correspondence should be addressed to L. Rueckert, Department of Psychology, Northeastern Illinois University, 5500 N. St. Louis Ave., Chicago, IL 60625 (e-mail: 1-rueckert@neiu.edu). pating. Such a requirement will likely partially eliminate the potential increase in sample size and diversity since many participants may be unwilling or unable to download the required plug-in (i.e., those who access the Internet using computers in public labs or libraries).

The purpose of the present study was to create a reliable measure of cerebral asymmetry using only HTML and JavaScript, which can be interpreted by the vast majority of Web browsers that are currently in use. We chose to use one of the simpler and well-studied lateralization tasks, the Levy chimeric faces task (Levy, Heller, Banich, \& Burton, 1983). In this task, participants view two chimeric faces (faces with one half smiling and the other half showing a neutral expression). The two faces are mirror images of each other and are aligned vertically on a single page. The participant is asked to judge which face, the top one or the bottom one, appears happier. Numerous studies have shown that right-handed participants are significantly more likely to choose the chimera with the smile to their (the viewers') left. This is thought to be due to the role of the right cerebral hemisphere $(\mathrm{RH})$ in the perception of emotion in faces. When asked to do the task, the participant's RH is activated, which causes a bias of attention to the left side of space, thereby making the chimera with the smile to the left appear happier than the one with the smile to the right. (For an extensive discussion of the design and rationale behind the study, see Levy et al., 1983.) This explanation of the leftward bias is supported by several studies in which lefthanders have shown a significantly reduced leftward bias in comparison with right-handers (Hellige et al., 1994; Hoptman \& Levy, 1988; Levy et al., 1983; Luh, Redl, \& Levy, 1994).

The leftward bias is highly reliable and has been found whether the stimuli are presented on a page or via a slide projector (Levy et al., 1983). Because a significant leftward bias can be obtained even when stimuli are presented in free vision, without control over viewing dis- 
tance or exposure duration, this task might be particularly well suited to the Web.

\section{METHOD}

\section{Task}

The task can be found at http://www.neiu.edu/ 1ruecker/ experiments. The Levy et al. (1983) chimeric faces task was replicated in a Web page using HTML and JavaScript. The original 36 stimuli were scanned and saved as .gif files. The presentation program was roughly based on a program described by Baron and Siepmann (2000). All participant responses were collected in a hidden window that was submitted only after all trials had been completed. A stimulus consisted of a new window that was meant to fill the entire screen. Within the window, the two chimeras were vertically aligned, centered, and sized to fill $70 \%$ of the height of the screen. For half of the trials, the chimera on the top had the smile to the left, and for the other half the chimera on the bottom had the smile to the left. The bottom chimera was always a mirror image of the top. Below the chimeras was the question, "Which face is happier?" Below that were radio buttons for "top" and "bottom." Below that was a button that said NEXT TRIAL. All of these were centered on the screen. In both Netscape and Internet Explorer (IE), the entire stimulus fit within the window, so no scroll bars were necessary.

Although the window in which the stimuli appeared was meant to fill the entire screen in order to make it perfectly symmetric (using the JavaScript "fullscreen" command), this was only possible for IE running under the Windows operating system. With Netscape for Windows, a title bar is included with the title at the upper-left corner of the screen. With both Netscape and IE for Macintosh, the menus remain on the screen in the upper-left corner. In addition, with Macintosh OS X, colored dots appear in the upperleft corner. Possible ramifications of this will be discussed in the Results section.

\section{Procedure}

All data were collected between April and October 2004. The participants first read a very brief description of the study. They were told that the experiment required the ability to use frames and JavaScript and would take considerably longer using a modem, so a T1 network, DSL, or cable connection was recommended. They were also told that, if they did not want their data to be analyzed for publication, they could type "demo" in the comment box at the end of the experiment. (The purpose of this was to allow other psychology researchers to assess the experiment without the participant actually being included in data analysis.) After this, the participants were linked to an informed consent form that gave more detailed information. At the bottom of the form, they could either click on "I consent to participate," in which case they were linked to the task instruction page, or they could click on "I do not consent," in which case they were linked back to the experiment main page.

In order to get an estimate of the number of participants who started the experiment but did not finish, the following information was submitted to the Northeastern Illinois University (NEIU) server, when the subject clicked on "I consent": IP address, operating system, browser name and version, date, and time.

After the participant clicked on "I consent," the instructions for the task were given, followed by the 36 stimuli described above. If the browser did not have JavaScript enabled, the stimuli were not presented and no responses were collected. For each pair of faces, the participants were asked to indicate which was happier, "top" or "bottom." If they clicked on "next trial" without making a choice, an alert appeared and they were prevented from advancing to the next trial. Pilot testing in our lab suggested that when utilizing a T1 or cable connection, the entire stimulus usually appeared within $1 \mathrm{sec}$. However, when utilizing a modem it could take up to $1 \mathrm{~min}$ to load. If the participant attempted to make a choice before the picture had fully loaded, an alert appeared.

After going through all 36 stimuli, a demographics form was presented asking questions about gender, handedness, birth date, race, history of neurological disorders, and so forth. In an attempt to identify multiple submissions from the same participants, it also asked if they had ever participated in this, or a similar, experiment previously. They could answer "yes," "no," or "not sure." The participants could also optionally give their name, e-mail address, and comments. Other researchers have suggested that personal information be gathered at the beginning of a Web study in order to minimize dropout (Reips, 2002). However, in the present study the demographics form was presented at the end because it is likely that the "high hurdle" would be going through the 36 face pairs.

Upon the participant clicking on the "submit" button, the data were submitted to a cgi program on the NEIU server. This program was a modified version of a program written by Birnbaum (2001) that put all data into a readable data file. If the participants gave their e-mail address, a receipt was sent to that address. (Some participants are motivated to get extra credit in psychology classes and require a receipt confirming their participation.) The following environmental variables were also automatically recorded: screen size, IP address, operating system, navigator name and version, date, start time (when the participant clicked on "start experiment"), and end time (when he or she clicked on "submit").

After submitting the data, the participant was told his or her number of smile-right choices, smile-left choices, and laterality quotient (LQ) ([right - left] / [right + left]). A negative LQ indicates that the participant chose the face with the smile to his or her left more often. The participants were then linked to a debriefing page that explained the purpose of the study and included links to other sites with information on neuropsychology and face processing.

\section{Participants}

The participants were recruited through signs placed on the NEIU Psychology Department bulletin boards. The Web site was also listed on search engines such as Yahoo and Google and on John Krantz's Psychological Research on the Net page (available at http:// psych.hanover.edu/research/exponnet.html).

Data submitted during pilot testing in our lab were identified by IP address and deleted. Of the 314 participants who submitted data, 20 were obvious duplicate submissions ( 2 or more cases with the same IP address submitted within a few minutes of each other, most likely due to clicking on "submit" more than once). In these cases, only data from the first submission were retained. Data from an additional 32 participants were deleted due to a total score (left choices + right choices) greater than 36 . (This can occur in some browsers if the participant goes back to a previous stimulus and gives a second response. After 3 weeks of testing, a note was added to the instruction page telling participants that they could not go back to previous stimuli. This reduced the problem substantially.) Because a response was required on every trial, the total could not be less than 36. Also deleted were data from participants who reported that they had, or might have, participated before $(n=27)$, who typed "demo" in the comment box $(n=1)$, did not report $20 / 20$ normal or corrected vision $(n=38)$, had a neurological disorder or suffered a head injury involving loss of consciousness $(n=$ $30)$, or did not report being left- or right-handed $(n=5)$. The final data set consisted of 161 participants. There were 40 men $(25 \%$ of the total sample; 33 right-handed and 7 left-handed) and 120 women (112 right-handed and 8 left-handed). One left-handed participant did not report gender. Men were significantly more likely to be left- 
handed than women $\left[\chi^{2}(1, N=161)=13.05, p=.001, \phi=.285\right]$. The reported ages ranged from 18 to 62 years, with a mean of 26.63 years. Two participants did not report their birthdate.

In the demographics form, the participants were asked to enter race in a text field. Based on the responses, they were categorized as Caucasian $(70 \%)$, African-American $(8 \%)$, Hispanic $(10 \%)$, Asian $(8 \%)$, or other $(4 \%)$.

Of the 161 participants providing usable data, $83 \%$ used IE for Windows as their browser (4\% Version 5.5, 79\% Version 6), 7\% used IE for Macintosh, $9 \%$ used Netscape for Windows (3\% Version 4.8, 6\% Version 5), and 1\% used Netscape for Macintosh. It must be noted that the browser version reported through the JavaScript command may not always accurately reflect the browser version used.

\section{RESULTS}

For right-handers, the mean LQ was -.463 , which is significantly less than $0[t(144)=-10.66, p=.000]$, indicating the expected leftward bias. The mean for lefthanders $(-.351)$ is also significantly less than $0[t(15)=$ $-2.14, p=.049]$. Both of these scores are substantially more negative than those obtained by Levy et al. (1983; see Figure 1), although only the right-handers' scores differed significantly [independent $t(254)=2.6, p<$ .01 , squared point-biserial correlation $\left.r_{\mathrm{pb}}^{2}=.026\right]$. The mean LQ in the Levy et al. study was -.303 for righthanders and -.134 for left-handers. Although the variance for LQ in right-handers in the present study (.266) was somewhat higher than that reported by Levy et al. (.194), the difference was not significant.

As expected, LQs were very reliable. The split-half reliability was .95 for right-handers and .97 for left-handers. These are very close to those reported by Levy et al. (1983), who obtained split-half reliabilities of .925 for both left- and right-handers. LQ was not related to gender, age, screen size, or time taken to complete the task.

\section{Browser and Operating System Effects}

One obvious possible explanation for the greater leftward bias observed in the present study, compared with the Levy et al. (1983) pencil-and-paper study, is the asymmetry of stimuli that occurred in some browsers. As described in the Method section, a perfectly symmetrical stimulus could be achieved only in IE running under the Windows operating system. In other browsers, there were additional stimuli in the upper-left corner, which may have biased participants' attention to the left. Although right-handed participants using IE for Windows did show less leftward bias than those using other browsers (IE for Windows LQ, $M=-.453$; other browsers, $M=$ $-.512)$, the difference did not approach significance $[t(143)=.498, p=.62]$. Furthermore, when only the 121 right-handed participants using IE for Windows are considered, they still show a significantly greater leftward bias than did participants in the Levy et al. (1983) study [independent $\left.t(230)=2.38, p<.05, r_{\mathrm{pb}}^{2}=.024\right]$. Due to the asymmetry of the screen in browsers other than IE for Windows, all data reported below include only participants using IE for Windows. In none of the analyses did this change the significance of the results.

\section{Time to Complete the Task}

The distribution of times taken to complete the task can be seen in Figure 2. Times ranged from a minimum of $3 \mathrm{~min}$ to a maximum of $38 \mathrm{~min}$, with a median of $7 \mathrm{~min}$. Eighty-five percent of all participants took $10 \mathrm{~min}$ or less, and $93 \%$ took less than $15 \mathrm{~min}$. To determine whether time taken to complete the task was related to LQ, Spearman's $\rho$ was calculated for right-handed participants using IE for Windows. The result was not significant $(\rho=.139, p=.130)$.

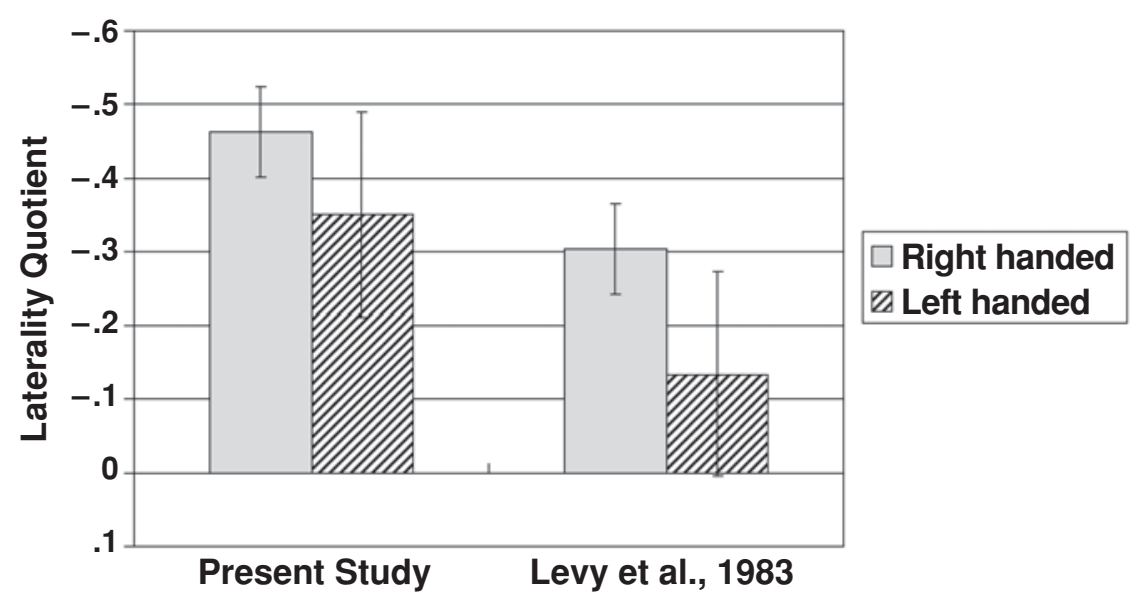

Figure 1. Laterality quotient for left- and right-handers in the present study, in comparison with the Levy et al. (1983) study (note the reversed scale with a higher bar indicating a greater leftward bias; error bars indicate the standard error of the mean for the independent $t$ test). 


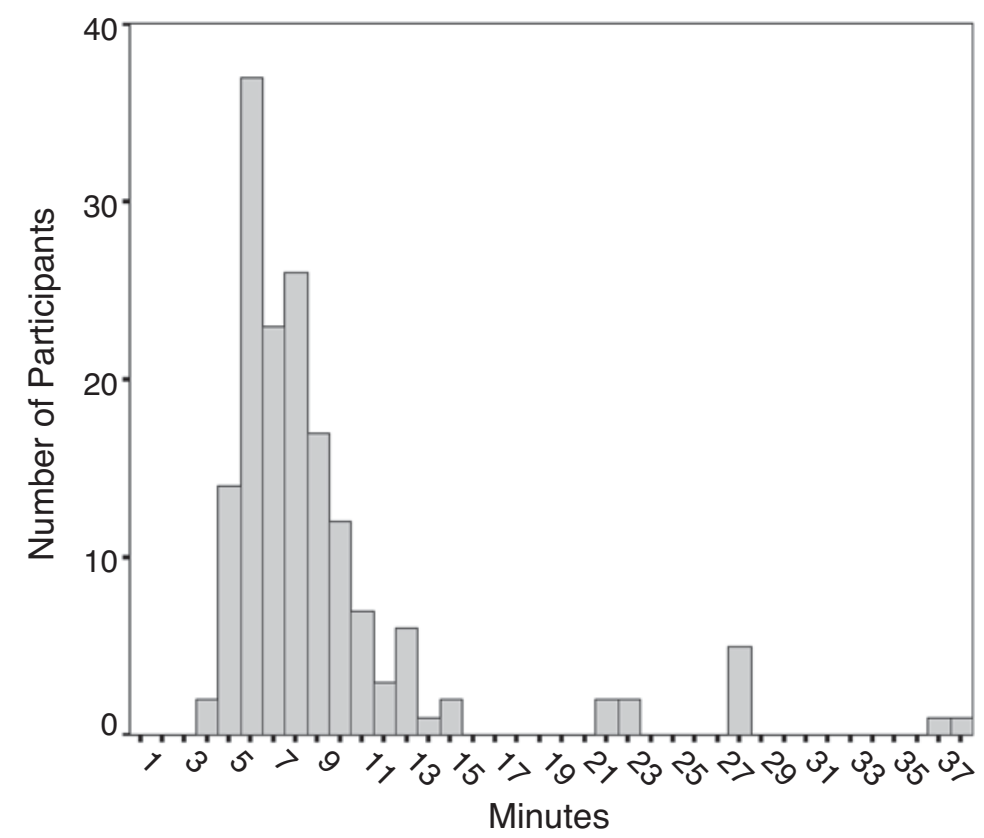

Figure 2. Distribution of time taken to complete the task.

\section{Race/Ethnic Group Effects}

Right-handed Caucasian participants obtained a significantly more left-biased LQ $(M=-.523)$ than did right-handed non-Caucasian participants $[M=-.312$; $\left.t(119)=2.14, p=.034, r_{\mathrm{pb}}^{2}=.037\right]$. Although all nonCaucasian participants were grouped together for this $t$ test due to small $n \mathrm{~s}$, Figure 3 shows that it was mainly African Americans, and to a lesser extent Asians, who differed from Caucasian participants.

\section{Dropouts}

After eliminating data submitted during pilot testing in our lab (identified by IP address), a total of 675 participants clicked on "I consent." This means that 362 responses to the consent form (about 53\%) did not continue through the whole study and submit data. Many of the 675 responses to the consent form consisted of more than one response from the same IP address, sometimes within a few minutes of each other. These are likely from participants who were experiencing technical difficulties with the task and tried more than once. There appeared to be no systematic differences between browsers. Unfortunately, there is no way to know at what point during the study these participants dropped out.

\section{DISCUSSION}

The results suggest that some measures of cerebral asymmetry can be reliably administered via the Web. Participants tested via the Web exhibited the expected leftward bias on a chimeric faces task. Right-handers chose the chimera with the smile to the left on $73 \%$ of trials, and left-handers chose the smile-left chimera on $67 \%$ of trials. Furthermore, the fact that the leftward bias observed in the present study was actually greater than that observed in other studies indicates that the many problems associated with increased variability in task administration (differences in computer screens, testing environment, etc.) did not adversely affect the measurement of asymmetry. However, the fact that the observed leftward bias was significantly stronger than the bias observed in more traditional pencil-and-paper studies indicates that the results must be interpreted with caution. Although the difference between the two studies was only significant for right-handed participants, the difference for left-handers was of equal magnitude and only failed to reach significance due to small $n$.

Although the data do not allow firm conclusions to be drawn regarding differences in browsers, it does appear that the lack of symmetry in some browser screens may affect the results. Nevertheless, this probably cannot entirely explain the difference between the Web-based results and those reported by Levy et al. (1983), since a significant difference remains when analyzing only participants using IE for Windows.

One difference between the original study by Levy et al. (1983) and the present study was the response requirement; participants in the former study were allowed to give an "I can't decide" response, but those in the latter study were not. This is also unlikely to explain the difference in results. In a previous study in our lab (Rueckert \& Pawlak, 2000), using the same chimeric faces task but requiring participants to make a choice on each trial yielded a mean LQ of -.220 for right-handers, which is actually less left-biased than that found by Levy et al.

One potentially troublesome explanation for the difference could be differential participant dropout. Some previous studies have suggested that a reduced leftward 


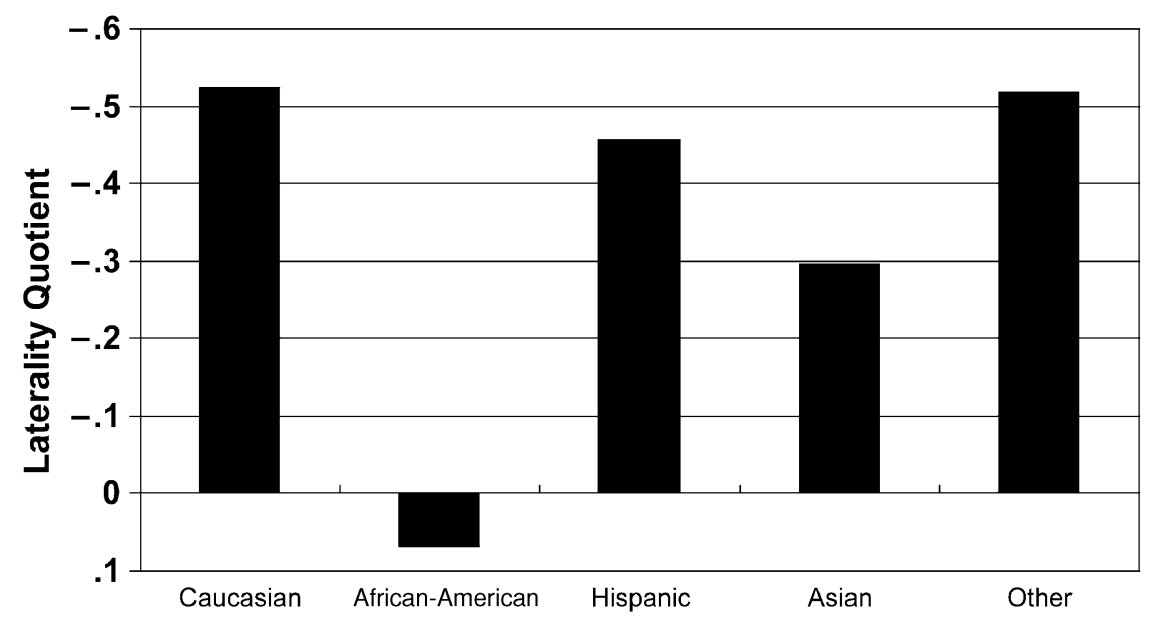

Figure 3. Laterality quotient by race.

bias is found on chimeric faces tasks when the discrimination between stimuli is more difficult (Carbary, Almerigi, \& Harris, 2001, 2002; see also the discussion of racial differences below). Although participants virtually never drop out during the course of the task in studies that take place in the lab, it is quite possible that the reduced social pressure to complete the task when performed on the Web would result in more dropping out after experiencing some, but not all, of the stimuli. It is also conceivable that participants who find the discrimination between the two faces more difficult would be more likely to discontinue participation than those who find it relatively easy. Unfortunately, this possibility cannot be tested, given the present results. However, this could easily be tested in the future by changing the task so that data are submitted more than once during the course of the experiment.

Despite a significant difference between the present results and the original study by Levy et al. (1983), it must be pointed out that the difference was very small, accounting for only $2.4 \%$ of the variance in LQ scores. Furthermore, it is possible that the observed difference can be attributed to systematic error in the pencil-andpaper version (Reips, 2000).

Web administration of this task shows great potential for future studies. The potential benefits of Web administration include increased sample size, increased sample diversity, and the ability to test participants with relatively rare neurological disorders. The importance of these factors is suggested by studies that have found that asymmetry scores on chimeric faces tasks are related to cognitive and personality measures (Rueckert \& Pawlak, 2000), depression (David \& Cutting, 1990; Jaeger, Borod, \& Peselow, 1987; Kucharska-Pietura \& David, 2003), and schizophrenia (David \& Cutting, 1990; KucharskaPietura, David, Dropko, \& Klimkowski, 2002).

In fact, a potentially very interesting topic for further study has already been suggested in the present results.
This is the first report of racial differences for this task. Most previous studies utilizing this task did not report the racial makeup of their sample, and none included race as an independent variable. The racial difference found in the present study was not completely unexpected. All of the faces included in the task were of Caucasian males. The fact that people are better at recognizing faces of members of their own race is well documented (for a review, see Meissner \& Brigham, 2001). It has been suggested that, when recognizing faces from their own race, participants rely mainly on configural information, but when recognizing less familiar faces, such as those of people of a different race, they attend more to individual features of the face (Diamond \& Carey, 1986; Rhodes, Brake, Taylor, \& Tan, 1989). This difference in processing strategy might well be expected to lead to a shift from RH to LH processing for faces of less familiar races. Of course, this effect will have to be replicated in future studies with more non-Caucasian participants and non-Caucasian chimeric faces before firm conclusions can be drawn. The use of the Web to administer this task would also make it relatively easy to include participants from a wide variety of foreign countries.

Because this task involved presentation of photographs, the time to complete the task could be relatively long for participants utilizing a modem. This is a potential problem, because those using a modem would see the stimulus drawn slowly, from top to bottom, which could alter the results. Although participants were not asked to report the method used for connecting to the Internet, inspection of Figure 2 shows that most participants completed the task within $15 \mathrm{~min}$. Since it generally took at least $30 \mathrm{sec}$, and often up to $1 \mathrm{~min}$, for a stimulus to load using a modem, it is unlikely that these participants were using a modem. This may be due to the warning, given on the main page participants saw before starting the task, that it would take much longer if a modem was used. Since the time to complete the task did not corre- 
late with LQ, it is unlikely that the type of Internet connection had an effect.

The chimeric faces task was specifically chosen for study via the Web because previous studies had suggested that very reliable asymmetry scores could be obtained with relatively little control over stimulus variables such as size, location, and exposure duration. Whether similar reliability can be achieved for studies requiring greater control (i.e., tachistoscopic studies measuring reaction time) is a question for future study. However, the results reported by McGraw et al. (2000) are very promising. The benefits of Internet testing may be less likely to outweigh the drawbacks for neuropsychological studies compared with social psychological studies, but for certain topics (i.e., the rapidly growing field of social neuroscience), the increase in sample size and diversity afforded by the Internet may well make it the method of choice for some studies.

\section{REFERENCES}

Baron, J., \& Siepmann, M. (2000). Techniques for creating and using Web questionnaires in research and testing. In M. H. Birnbaum (Ed.), Psychological experiments on the Internet (pp. 235-265). San Diego: Academic Press.

BIRnbaum, M. H. (2001). Introduction to behavioral research on the Internet. Upper Saddle River, NJ: Prentice-Hall.

Carbary, T. J., Almerigi, J. B., \& Harris, L. J. (2001). The left visual hemispace bias for the perception of chimeric faces: A further test of the difficulty-of-discrimination hypothesis. Brain \& Cognition, 46, 57-62.

Carbary, T. J., Almerigi, J. B., \& Harris, L. J. (2002). Task difficulty reduces the left visual hemispace bias for judgments of emotion in chimeric faces. Brain \& Cognition, 48, 304-311.

David, A. S., \& CutTing, J. C. (1990). Affect, affective disorder and schizophrenia: A neuropsychological investigation of right hemisphere function. British Journal of Psychiatry, 156, 491-495.

DiAmond, R., \& CAREY, S. (1986). Why faces are and are not special: An effect of expertise. Journal of Experimental Psychology: General, 115, 107-117.

Gosling, S. D., Vazire, S., Srivastava, S., \& John, O. P. (2004). Should we trust Web-based studies? A comparative analysis of six preconceptions about Internet questionnaires. American Psychologist, 59, 93-104.
Hellige, J. B., Bloch, M. I., Cowin, E. L., Eng, T. L., Eviatar, Z., \& SERGENT, V. (1994). Individual variation in hemispheric asymmetry: Multitask study of effects related to handedness and sex. Journal of Experimental Psychology: General, 123, 235-256.

Hoptman, M., \& Levy, J. (1988). Perceptual asymmetries in left- and right-handers for cartoon and real faces. Brain \& Cognition, 8, 178-188.

JAeger, J., Borod, J. C., \& Peselow, E. (1987). Depressed patients have atypical hemispace biases in the perception of emotional chimeric faces. Journal of Abnormal Psychology, 96, 321-324.

Kraut, R., Olson, J., Banaji, M., Bruckman, A., Cohen, J., \& Couper, M. (2004). Psychological research online: Report of Board of Scientific Affairs advisory group on the conduct of research on the Internet. American Psychologist, 59, 105-117.

Kucharska-Pietura, K., \& David, A. S. (2003). The perception of emotional chimeric faces in patients with depression, mania and unilateral brain damage. Psychological Medicine, 33, 739-745.

Kucharska-Pietura, K., David, A. S., Dropko, P., \& KlimkowSKI, M. (2002). The perception of emotional chimeric faces in schizophrenia: Further evidence of right hemisphere dysfunction. Neuropsychiatry, Neuropsychology, \& Behavioral Neurology, 15, 72-78.

Levy, J., Heller, W., Banich, M., \& Burton, L. (1983). Asymmetry of perception in free viewing of chimeric faces. Brain \& Cognition, 2, 404-419.

LuH, K., ReDL, J., \& LeVy, J. (1994). Left- and right-handers see people differently: Free-vision perceptual asymmetries for chimeric stimuli. Brain \& Cognition, 25, 141-160.

McGraw, K. O., Tew, M. D., \& Williams, J. E. (2000). The integrity of Web-delivered experiments: Can you trust the data? Psychological Science, 11, 502-506.

Meissner, C. A., \& Brigham, J. C. (2001). Thirty years of investigating the own-race bias in memory for faces: A meta-analytic review. Psychology, Public Policy, \& Law, 7, 3-35.

REIPS, U.-D. (2000). The Web experiment method: Advantages, disadvantages, and solutions. In M. H. Birnbaum (Ed.), Psychological experiments on the Internet (pp. 89-117). San Diego: Academic Press.

REIPS, U.-D. (2002). Standards for Internet-based experimenting. Experimental Psychology, 49, 243-256.

Rhodes, G., Brake, S., Taylor, K., \& Tan, S. (1989). Expertise and configural coding in face recognition. British Journal of Psychology, 80, 313-331.

RUECKERT, L., \& PAWLAK, T. (2000). Individual differences in cognitive performance due to right hemisphere arousal. Laterality, 5, 77-89.

(Manuscript received November 15, 2004; revision accepted for publication May 27, 2005.) 